\title{
Search for black hole ringdown signals in LIGO S4 data
}

\author{
Lisa M Goggin (for the LIGO Scientific Collaboration) \\ California Institute of Technology, M/C 18-34, Pasadena, CA 91125, USA \\ E-mail: lgoggin@ligo.caltech.edu
}

Received 31 March 2006, in final form 14 June 2006

Published 15 September 2006

Online at stacks.iop.org/CQG/23/S709

\begin{abstract}
If a coalescing binary system results in a black hole we expect it to be a perturbed Kerr black hole and to radiate gravitational waves in the form of ringdowns. A search for such signals in data from the fourth LIGO science run is currently being developed. In this paper we outline the theory on which this search is based and use it to predict the range for this data set.
\end{abstract}

PACS numbers: 95.85.Sz, 04.80.Nn, 07.05.Kf, 97.60.Lf

\section{Introduction}

The final state of a compact binary coalescence is expected to be a perturbed black hole which will radiate away the perturbations as gravitational waves. At late times, the distorted black hole will be sufficiently similar to a Kerr black hole [1] that black hole perturbation theory can be used to model the waveforms [2]. These ringdown waveforms are a superposition of quasi-normal modes, the dominant of which is expected to be a spherical harmonic of spin weight 2 . The frequency and damping time uniquely depend on the mass and spin of the black hole. This uniqueness is directly related to the no-hair theorem and a reliable detection of ringdown waveforms would provide a key test of general relativity in the strong field regime [3].

\section{Quasi-normal modes}

These waveforms can be searched for using the method of matched filtering [4], that is, looking for correlations between the noisy detector output and a set of waveform templates.

The template used to search for ringdowns in data from the fourth LIGO science run (S4) is a damped sinusoid with characteristic frequency $f_{0}$ and quality factor $Q$ given by

$$
h\left(t-t_{0}\right)=A \mathrm{e}^{-\frac{\pi f_{0}}{Q} t} \cos \left(2 \pi f_{0} t+\phi_{0}\right)
$$




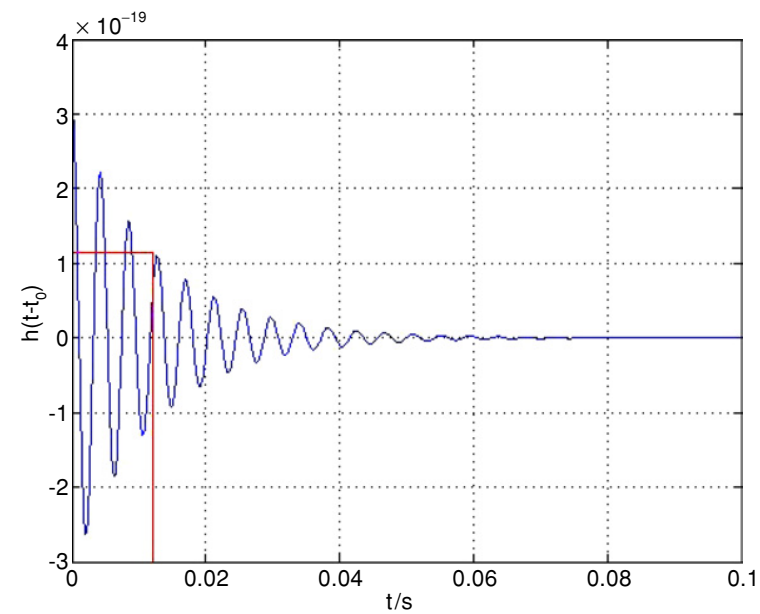

Figure 1. Ringdown waveform from a black hole of mass $100 M_{\odot}$ and spin 0.96 corresponding to (see the text) $f_{0}=235 \mathrm{~Hz}$ and $Q=9$, at a distance of $1 \mathrm{Mpc}$. To illustrate the short nature of these waveforms the e-folding time is also marked.

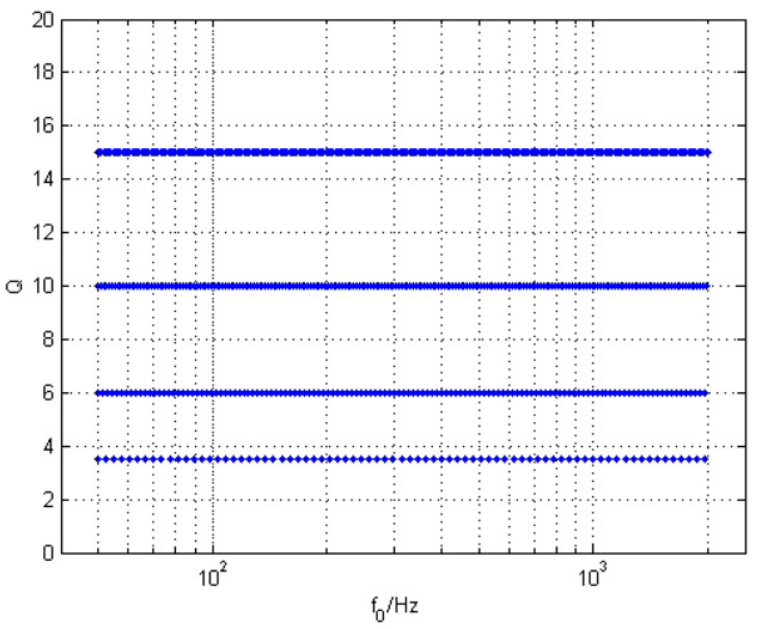

Figure 2. An example of the template bank for the S4 ringdown search.

The other unknown parameters, amplitude $A$, time of arrival at the detector $t_{0}$ and initial phase $\phi_{0}$, can be found using standard matched-filtering techniques. An example of a ringdown waveform is shown in figure 1 .

Following the method of Owen [5], Creighton [6] has laid out the ringdown template bank according to the metric

$$
\mathrm{d} s^{2} \approx \frac{1}{8} \frac{\mathrm{d} Q^{2}}{Q^{2}}-\frac{1}{4} \frac{\mathrm{d} Q}{Q} \frac{\mathrm{d} f_{0}}{f_{0}}+Q^{2} \frac{\mathrm{d} f_{0}^{2}}{f_{0}^{2}},
$$

where $\mathrm{d} s$ is the mismatch (loss of signal to noise ratio, SNR) between templates. This method modifies equation (2) by taking the noise power spectral density of the data segment being 


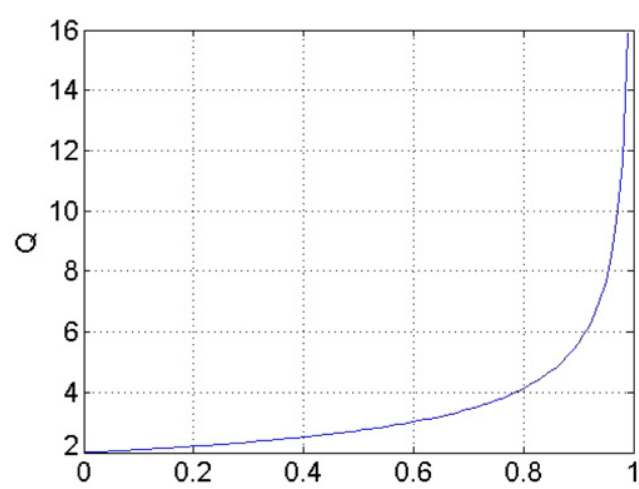

(a)

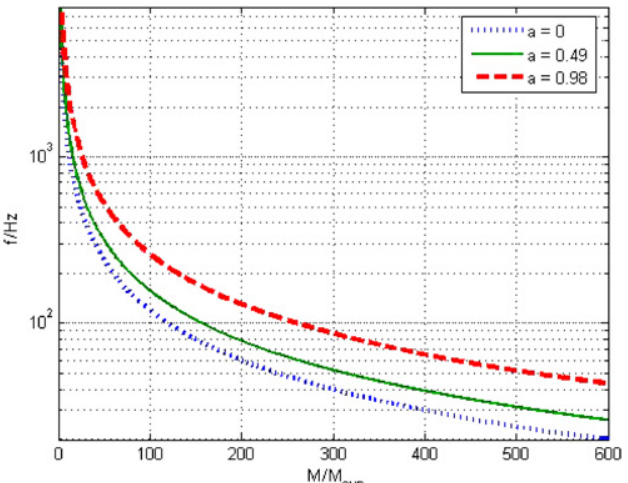

(b)

Figure 3. (a) Quality factor versus dimensionless spin parameter, $(b)$ frequency versus mass for $a=0,0.49,0.98$.

analysed into account. The number of templates generated per segment depends on the range of $f_{0}$ and $Q$ and for the search discussed below is of order 500. An example of the template bank layout is shown in figure 2 .

In searching the $\mathrm{S} 4$ data we require that the loss of SNR between a waveform and the nearest template be no more than 3\%. The frequency range for the LIGO S4 template bank is $50 \mathrm{~Hz}$ to $2 \mathrm{kHz}$ and that for $Q$ is 2 to 20 . The $f_{0}$ range is motivated by the detector sensitivity and the $Q$ range was chosen to sensibly encompass the spin range (discussed further below).

Determining the waveform parameters is useful only if they can be related to astrophysical quantities. Detweiler [7] showed that for the dominant mode $f_{0}$ and $Q$ are unique and invertible functions of the black hole's parameters, mass $M$ and dimensionless spin factor $a$. Using numerical computations by Leaver [8], Echeverria [9] formulated analytic expressions for $\left(f_{0}, Q\right)$ in terms of $(M, a)$ as follows:

$$
Q \approx 2(1-a)^{-0.45}
$$

and

$$
f_{0} \approx \frac{c^{3}}{2 \pi G M}\left[1-0.63(1-a)^{0.3}\right]
$$

where $a$ is defined in terms of the spin angular momentum, $a=S c / G M^{2}$, and ranges from 0 (Schwarzschild limit) to 1 (extreme Kerr limit). Using (3) and (4), we can express the template bank scope in terms of $M$ and $a ; 14<M / M_{\odot}<236$ and $0<a<0.994$. Figure 3(a) displays the dependence of $Q$ on $a$ and figure $3(b)$ shows how $f_{0}$ varies with $M$ for a fixed $a$.

To measure the efficiency of the search we simulate a population and inject it into the data stream. In doing this we have made some assumptions. The first is that $1 \%$ of the mass of the black hole is radiated as gravitational waves. Secondly, we define two orthogonal polarizations $h_{+}$and $h_{\times}$as

$$
h_{+}=h_{0}\left(1+\cos ^{2} \iota\right) \mathrm{e}^{-\frac{\pi f_{0}}{Q} t} \cos \left(2 \pi f_{0} t-\phi_{0}\right)
$$

and

$$
h_{\times}=h_{0}(2 \cos \iota) \mathrm{e}^{-\frac{\pi f_{0}}{Q} t} \sin \left(2 \pi f_{0} t-\phi_{0}\right),
$$

where $\iota$ is the angle of inclination. 


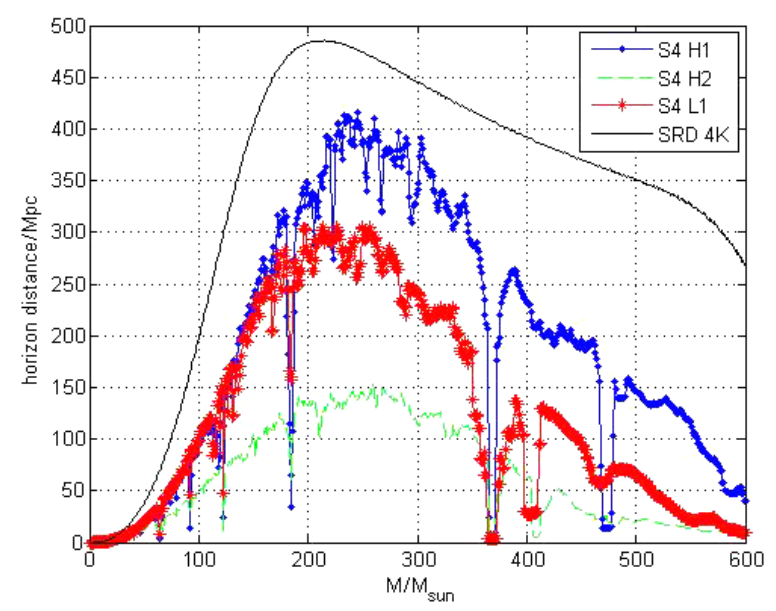

Figure 4. The horizon distance of the three LIGO detectors (the $4 \mathrm{~km}$ detector at Hanford, H1, the $2 \mathrm{~km}$ detector at Hanford, H2, and the $4 \mathrm{~km}$ detector at Livingston, L1) and the LIGO noise goal, $\mathrm{SRD}$, for a black hole with $a=0.9$ and $S N R=8$.

The signal we inject, $h(t)$, is a superposition of these two polarizations on the detector beam-pattern functions $F_{+}$and $F_{\times}$(which depend on the positon of the sources $(\alpha, \delta)$ and polarization $\Psi$ ),

$$
h(t)=h_{+}\left(t, \iota, \phi_{0}\right) F_{+}(\alpha, \delta, \Psi)+h_{\times}\left(t, \iota, \phi_{0}\right) F_{\times}(\alpha, \delta, \Psi) .
$$

Our simulated population is uniform in $M, a, \log ($ distance) and angles (mentioned above). These signals are injected into the data stream which is then passed through the ringdown search code. Examination of the output enables us to tune the search parameters.

A useful indicator of the sensitivity of the search is the 'horizon distance', or the maximum distance at which gravitational waves from an optimally oriented source with an SNR of 8 can be detected. Using the 'typical' S4 noise curves [10], we can plot this quantity for a source with a spin factor of $a=0.9$ and as a function of mass. As can be seen from figure 4, the S4 noise curves result in peak horizon distances for black holes of mass $\sim 236 M_{\odot}$, which can be detected to a distance of approximately $140 \mathrm{Mpc}$ for the LIGO $2 \mathrm{~km}$ detector at Hanford (H2), $300 \mathrm{Mpc}$ for the LIGO $4 \mathrm{~km}$ detector at Livingston (L1) and $400 \mathrm{Mpc}$ for the $4 \mathrm{~km}$ detector at Hanford (H1).

\section{Conclusion}

A first-pass filtering of the data has been completed and the parameters of the pipeline are currently being tuned using software injections of simulated ringdown signals from the astrophysical population described above. Multi-detector coincidence code is being developed which will help reduce the false alarm rate due to instrumental glitches. We expect to present a result for this search in the summer of 2006. Work is in progress to combine the ringdown search with that for the inspiral and merger phases.

\section{Acknowledgments}

The author gratefully acknowledges the support of the United States National Science Foundation for the construction and operation of the LIGO Laboratory and the Particle Physics 
and Astronomy Research Council of the United Kingdom, the Max Planck Society and the State of Niedersachsen/Germany for support of the construction and operation of the GEO600 detector. The author also gratefully acknowledges the support of the research by these agencies and by the Australian Research Council, the Natural Sciences and Engineering Research Council of Canada, the Council of Scientific and Industrial Research of India, the Department of Science and Technology of India, the Spanish Ministerio de Educación y Ciencia, the John Simon Guggenheim Foundation, the Leverhulme Trust, the David and Lucile Packard Foundation, the Research Corporation and the Alfred P Sloan Foundation.

\section{References}

[1] Kerr R P 1963 Phys. Rev. Lett. 11237

[2] Teukolsky S A 1973 Astrophys. J. 185646

[3] Dreyer O et al 2004 Class. Quantum Grav. 21787

[4] Wainstein L A and Zubakov V D 1962 Extraction of Signals from Noise (Englewood Cliffs, NJ: Prentice-Hall)

[5] Owen B J 1996 Phys. Rev. D 536749

[6] Creighton J D E 1999 Phys. Rev. D 60022001

[7] Detweiler S L 1980 Astrophys. J. 239292

[8] Leaver E W 1986 Proc. R. Soc. A 402285

[9] Echeverria F 1989 Phys. Rev. D 403194

[10] S4 Run Sensitivities, http://www.ligo.caltech.edu/lazz/distribution/LSC_Data/ 